\title{
GTND Giomaled

\section{II progetto di un fumetto nelle scuole per la cultura della donazione}

Giornale di Tecniche Nefrologiche e Dialitiche 2019, Vol. 31(2) 146-152

(C) The Author(s) 2019

Article reuse guidelines:

sagepub.com/journals-permissions

DOI: $10.1177 / 0394936219846822$

journals.sagepub.com/home/gtn

\section{@SAGE}

\section{Battista Catania}

\section{Carissimo Editor,}

sono un medico nefrologo di periferia che partecipa alle disavventure e alle gioie dei pazienti con malattia renale cronica, perché nel nostro Centro viviamo come una piccola grande famiglia dove il sorriso e una mano tesa non mancano mai.

Proprio l'opportunità di condividere a fianco dei malati l'iter trapiantologico e l'esplosione di energia dopo il trapianto $\mathrm{mi}$ ha portato a raccogliere in un libro la storia vera di un paziente che è stata poi trasformata in fumetti con l'obiettivo di parlare di donazioni nelle scuole.

Il trapianto renale presuppone una decisione anteriore, esplicita, libera e consapevole da parte del donatore: essere unico e speciale.

Si offre senza ricompensa una parte di sé per la salute e il benessere di una persona sconosciuta in difficoltà.

È un dono di amore, comunione, solidarietà che fa rivivere un'altra persona che rinasce:

con la donazione di organi si diventa supereroi perché pur morendo si rivive in un altro essere umano!

Le persone che non danno saranno sole ed egoiste: GREPPER E GRANNETTE attaccate alla "greppia" ma alla fine saranno dimenticate perché "polvere qualsiasi".

L'opposizione dei familiari all'espianto degli organi "post-mortem" ha rappresentato in Italia nel 2017 il 28\% delle cause di rifiuto e di non trapianto. Questo può essere evitato con un consapevole assenso rappresentato dalla firma del "testamento umano" in vita.

Bisogna vincere le paure e la mancanza di conoscenze, anche la Chiesa Cattolica considera un atto di amore e di generosità la donazione di organi post-mortem.
L'evento conclusivo della nostra vita interrompe drasticamente $\mathrm{i}$ forti rapporti umani consolidati in vita ma la possibilità di aiutare qualcuno in difficoltà può e deve alleviare la perdita.

Quando abbiamo operato tutto il possibile per curare un malato, davanti alla "sorella morte" inesorabile e "livella" per tutti, non resta che pensare oltre.

Il vantaggio che può determinare l'espianto di organi è tangibile con la medicina narrativa che tocca le corde dell'animo umano stimolando la riflessione e l'empatia.

Il futuro inizia con un'idea, un pensiero anche semplice che può cambiare il mondo.

Facciamo incontrare "chi dà e può dare" con "chi ha avuto" perché l'esperienza vissuta trasformata in fumetti per parlare ai giovani, diventa testimonianza tangibile e spinta emotiva a dare. Tutto ciò per realizzare un futuro migliore per le persone che soffrono e sopravvivono in dialisi, perché il trapianto ridona la vita e la vita è meravigliosa, un dono che può essere donato!

Con la collaborazione fra gli uomini queste idee, queste opportunità potranno crescere e fiorire perché diventeranno parte della cultura e della mentalità degli uomini del domani.

La generosità si apprende anche attraverso le storie che descrivono persone che agiscono in modo generoso: se poi si parla ai giovani è meglio raccontarle in fumetti.

\section{U.O. s DIALISI - Ospedale F. Lotti, Pontedera}

\section{Corrispondenza:}

Battista Catania, U.O. s DIALISI - Ospedale F. Lotti, Pontedera, Italy. Email: battista.catania@usInordovest.toscana.it 


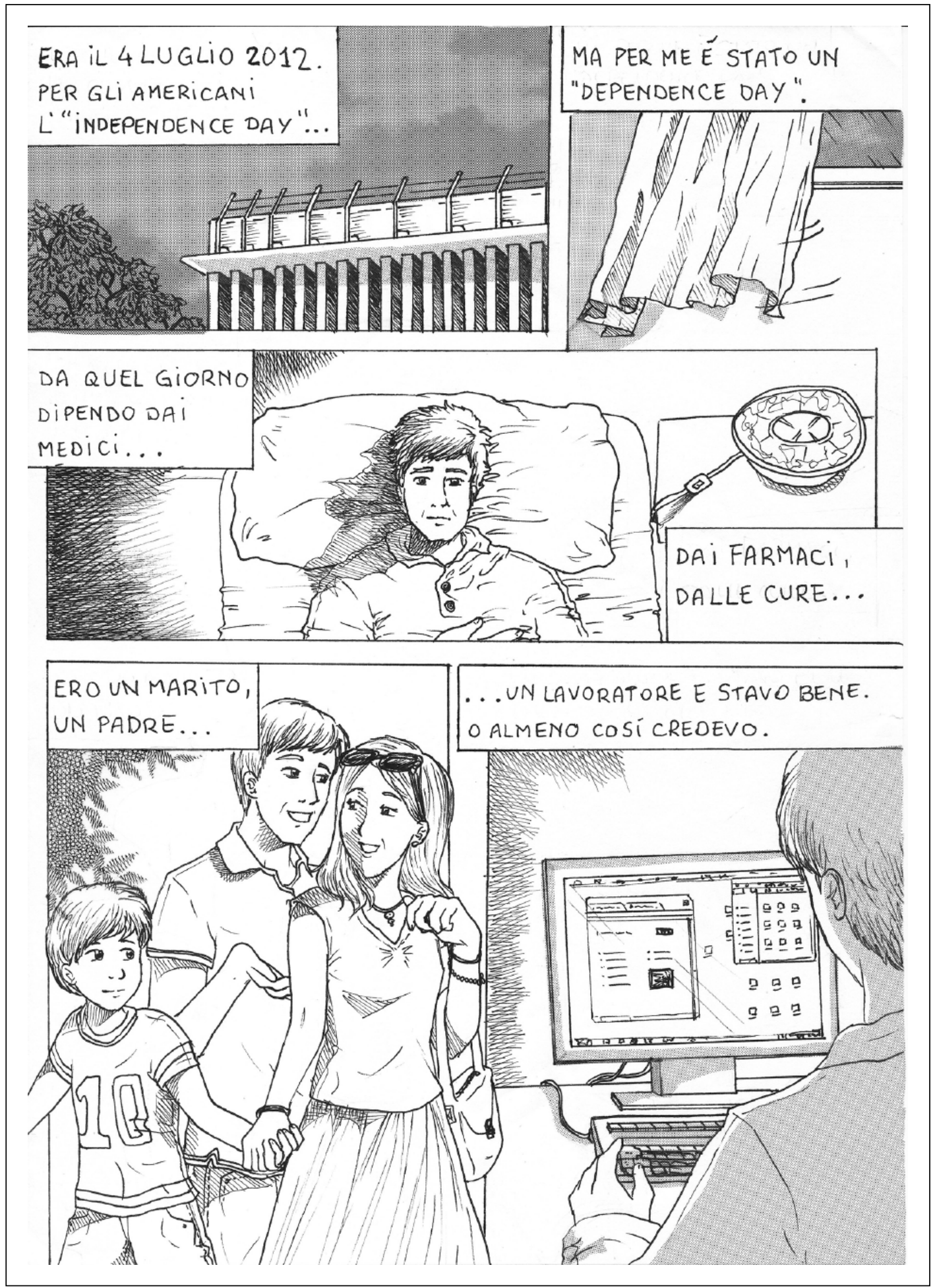

Figura 1. 


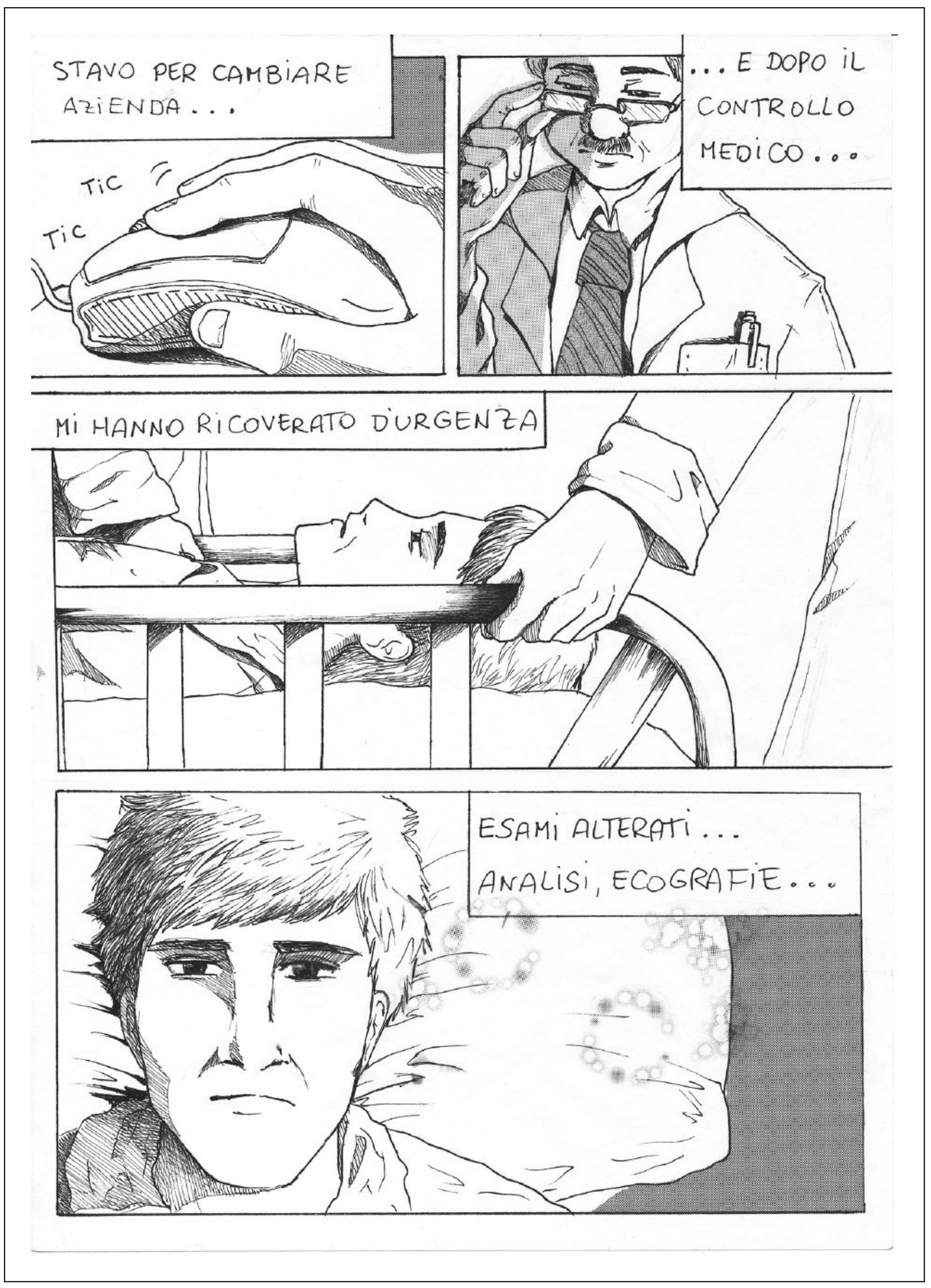

Figura 2. 


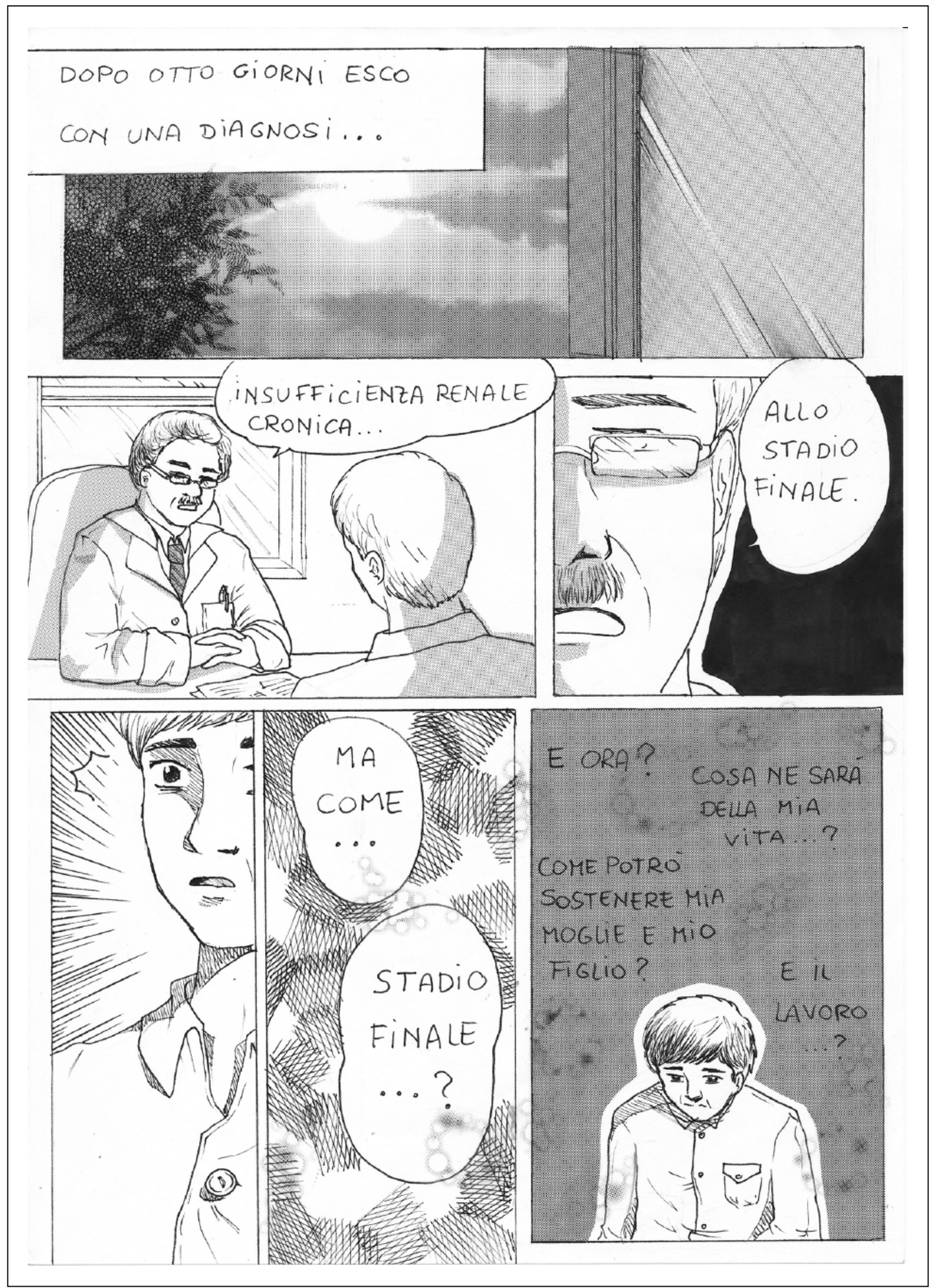

Figura 3. 


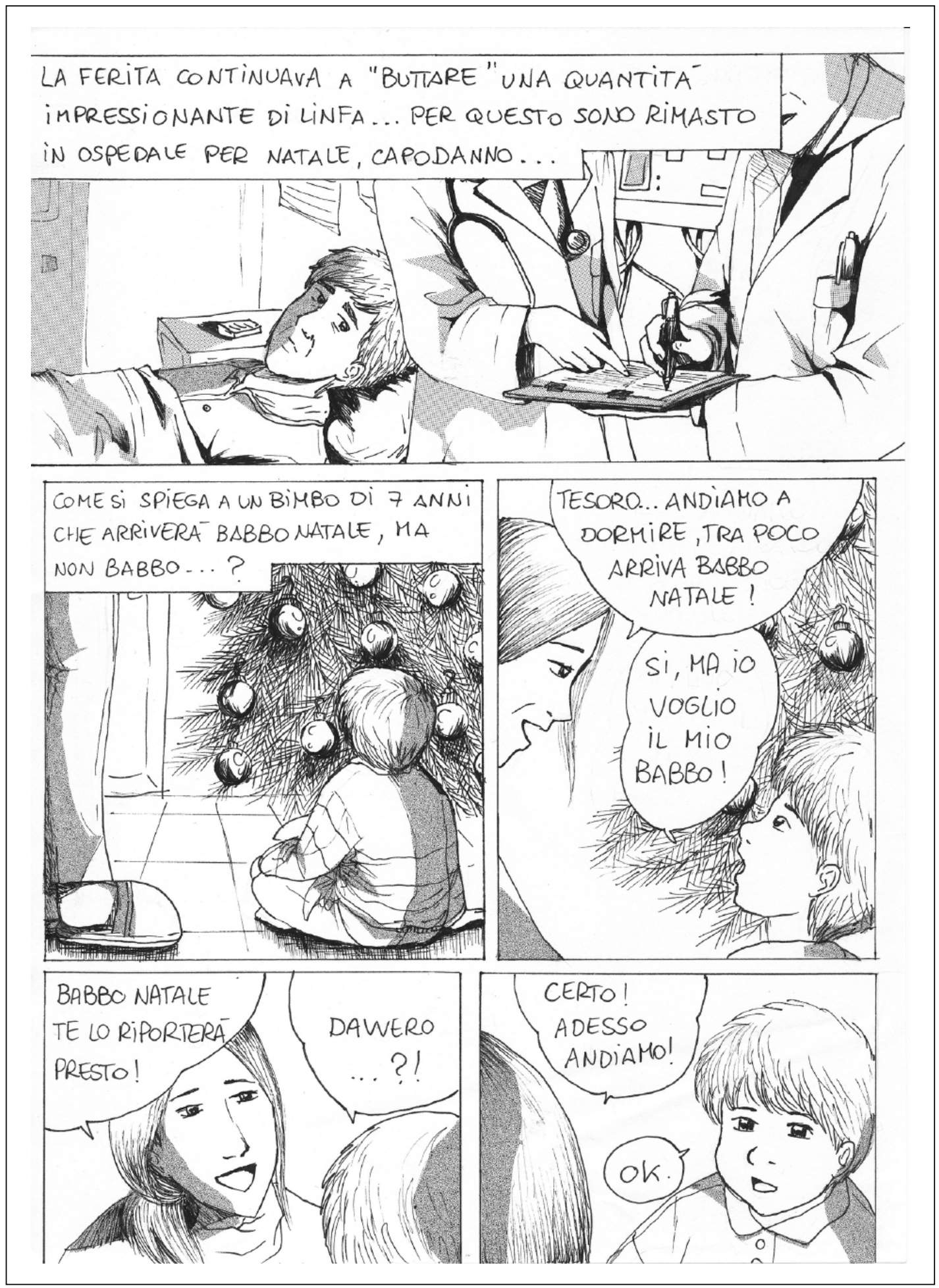

Figura 4. 


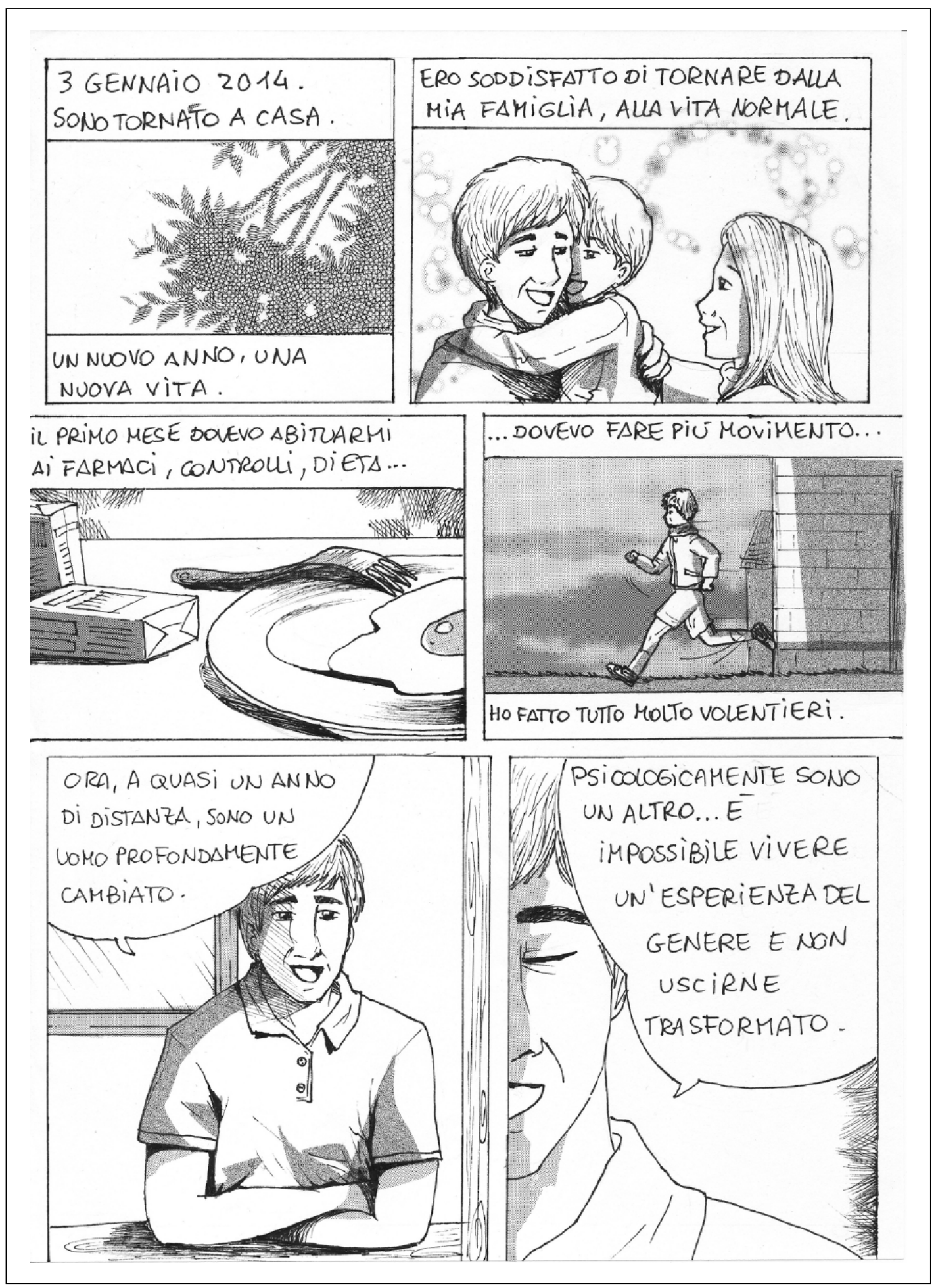

Figura 5. 


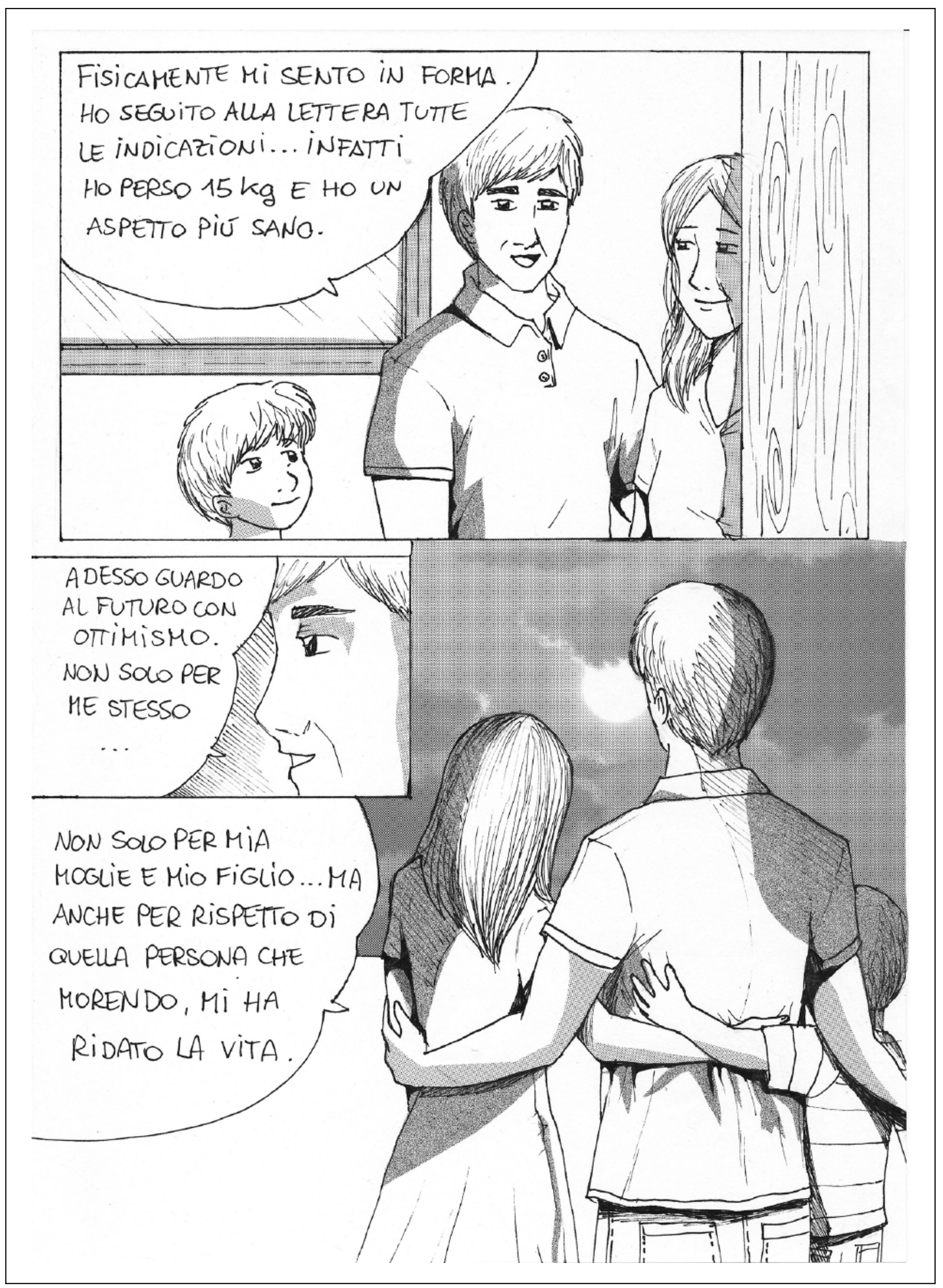

Figura 6. 